\title{
Research on Segmentation Production Simulation Optimization Technology Based on Plant Simulation
}

\author{
Xincheng Ma, Guoqing Zhang, Chong Zhang
}

\begin{abstract}
With the rapid development of science and technology, China's shipbuilding industry has also been rapidly improved. After several generations of ship industry elites' unremitting efforts, China's shipbuilding industry has entered the ranks of the world's shipbuilding industry. However, there is still a certain gap in production efficiency compared with Japan and South Korea. The main difference lies in the waste of invalid time on the segment production process. In the process of ship segmentation, there are often production waiting and backlog phenomena, which lead to bottlenecks on the production line. Therefore, this paper uses Plant Simulation to model and simulate the segment production line and optimize the production line by using the experimental design method to reduce Station blocking rate, shortening the ship construction period.
\end{abstract}

Index Terms - Plant Simulation; simulation modeling; segmentation production.

\section{INTRODUCTION}

Since shipbuilding is a very large system, the construction level is extremely complex, and planning management involves all aspects, effective management and control are particularly important. With the wide application of the pull production system in ship production, the virtual optimization simulation technology has become an important means in the lean production management of ships, and plays a vital role in the timely production of ships[1]. As a whole, the application of virtual simulation technology in shipbuilding mainly focuses on visual assembly and structural design. How to use simulation technology to realize the regulation of ship construction plan will become one of the important goals of digital shipbuilding[2]. To this end, this paper takes the ship segmentation manufacturing as the object, builds the production pipeline model, uses Plant Simulation to simulate the segmentation production line, finds the blocking station and uses the experimental design method to optimize the production line, thus reducing the station blocking rate. Shorten the ship construction cycle, increase the production efficiency of the shipyard and shorten the production cost.

\section{SEGMENTED PRODUCTION LINE PROCESS ANALYSIS}

Ship segmentation can be divided into plane linear segment, plane curve segment, curved segment, special segment, semi-stereo segment and solid segment according to its appearance characteristics, but there is no actual ship

Ma Xincheng, Student at Jiangsu University of Science and Technology. Graduate degree. Research direction for ship design and manufacturing

Zhang Guoqing, Student at Jiangsu University of Science and Technology. Graduate degree. Research direction for ship design and manufacturing

Zhang Chong, Student at Jiangsu University of Science and Technology. Graduate degree. Research direction for ship design and manufacturing production process[3]. It is divided into such fine parts, but it is divided into plane segments and surface segments according to its structural characteristics. In the staged production process, the segmented production process mainly includes three parts: component/frame assembly, panel welding and internal structure assembly; in its production organization, the segmentation production process can be divided into fixed stations. Production and assembly line production.

Plane segmentation is the simplest segment construction in ship construction both in structure and in production. The production process is simple and clear, which is very suitable for assembly line operation[4]. Although the surface segmentation structure is complex with respect to the plane segmentation structure, the segmentation structure of different ships is very different. However, after detailed analysis, it can be seen that the components, construction processes and production processes of all segments are basically the same. Design the assembly line.

In actual ship production, the shipyard will select the assembly line according to its own needs, and will change it according to the size of the shipyard and the equipment resources, so that it can better adapt to the actual production needs of the shipyard and make the best use of the assembly line. Complete more production work.

This paper summarizes the segmentation production line shown in Figure 1 according to the segmentation production process of a shipyard.

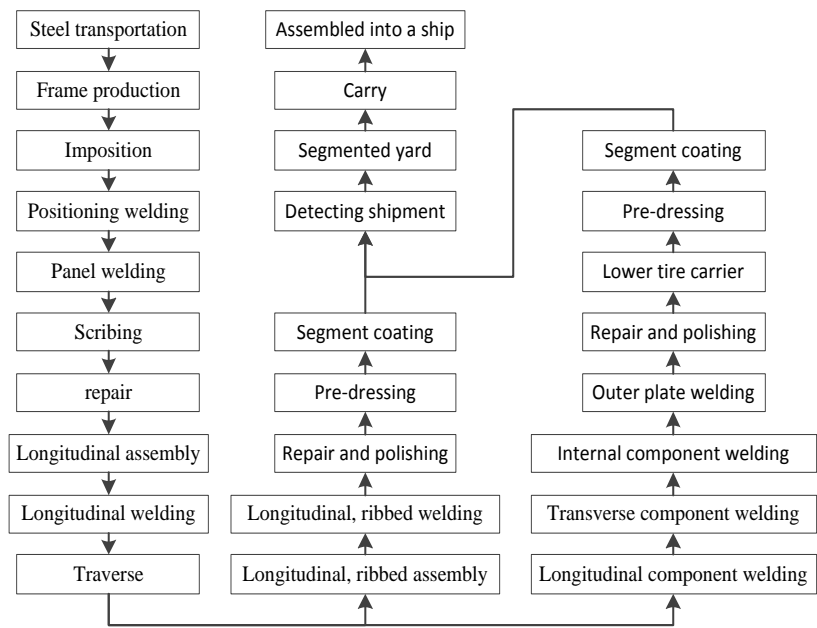

Fig. 1 Segmentation production process of a shipyard

\section{SELECTION OF SiMULATION SOFTWARE}

Discrete event systems have a large number of random variables. For example, the "transportation" time and "processing" time of products are random variables and contain complex logical relationships. Currently, for the 
characteristics of discrete systems, the academic community has adopted dozens of different modeling. Simulation software, application scope and purpose are different. The software used in this simulation modeling is Plant Simulation, an object-oriented discrete time dynamic simulation software, which is one of the best solution software for simulation and optimization in factories, production lines and production logistics[5]. The controls in Plant Simulation can be divided into two categories, one is physical controls, which are used to simulate real-world tools and devices; the other is data information processing control, which is used to input, analyze or control simulation controls. The corresponding controls and meanings used in this simulation are shown in Table 1.

Tab. 1 Names and meanings of the controls (See the appendix for details.)

\section{CReate A Simulation MODEL}

The Plant Simulation production system simulation software was used to simulate and simulate the ship in a shipyard with a ship number L0030. The specific model is shown in Figure 2.

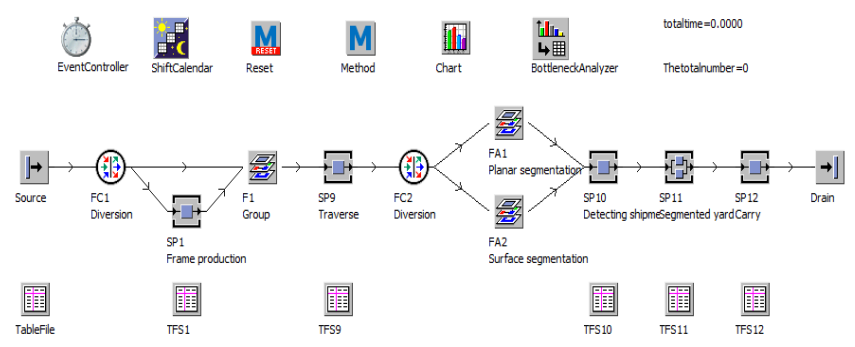

Fig. 2 Simulation model of a shipyard segment production process

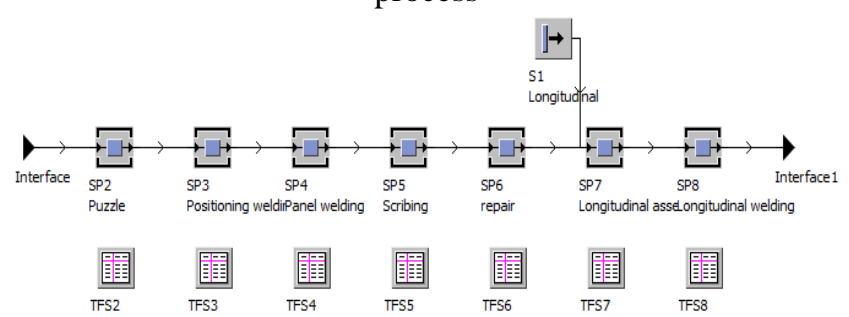

Fig. 3 Two-layer structure F1
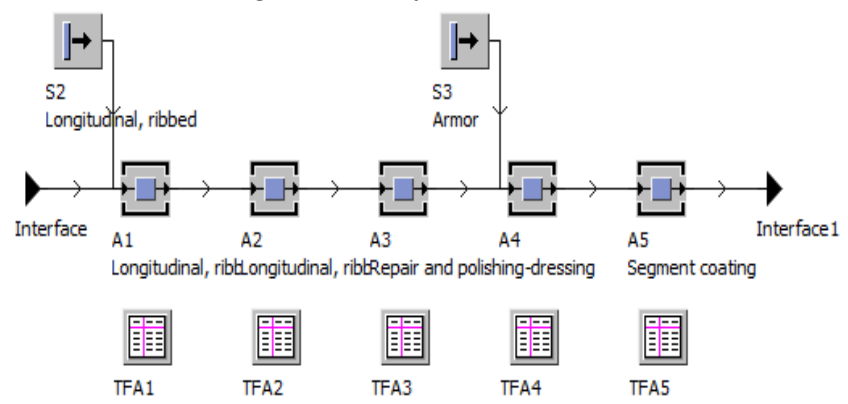

Fig. 4 Two-layer structure FA1

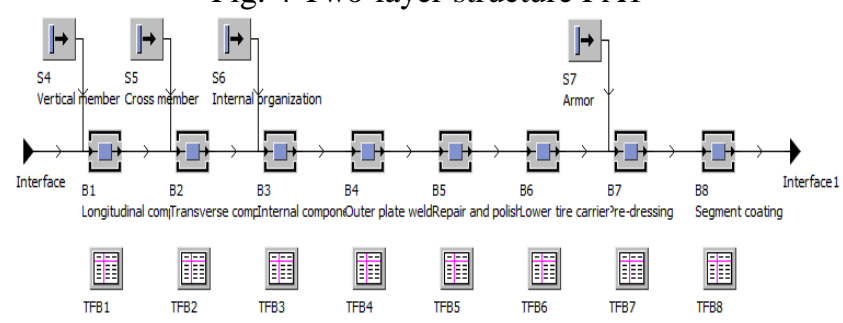

Fig. 5 Two-layer structure FA2
The ShiftCalendar control controls the production schedule of the employees. The shipyard stipulates that each work week is a single break, and the work hours are from Monday to Saturday. The daily work hours are from 7:00 to 12:00 and from 14:00 to 18:00. The middle 9:15-9:45 and 15:45-16:15 are halfway breaks. By programming the Method, the pipeline is controlled accordingly, and the final production time and the production efficiency of each station are obtained. By editing the Table File, the processing time of each station in the production process is set in detail.

\section{Simulation RESUltS}

The production data of the 44 segments of the ship was brought into the model for simulation, and some of the data are shown in Table 2 below.

Tab. 2 Sectional production data for a type of ship

(See the appendix for details.)

Through the simulation of the model, we can get the total time required for the production of 44 segments is 52:12:59:21, and the production efficiency of each station is shown by the statistical chart as shown in Figure 6. It can be seen from the statistical graph that there is still a large blockage in the segmentation production process, especially the SP2-SP8 station has a serious blockage. It will definitely affect the ship's production efficiency and increase the total production time. Therefore, it is necessary to properly adjust and optimize the segmentation production process.

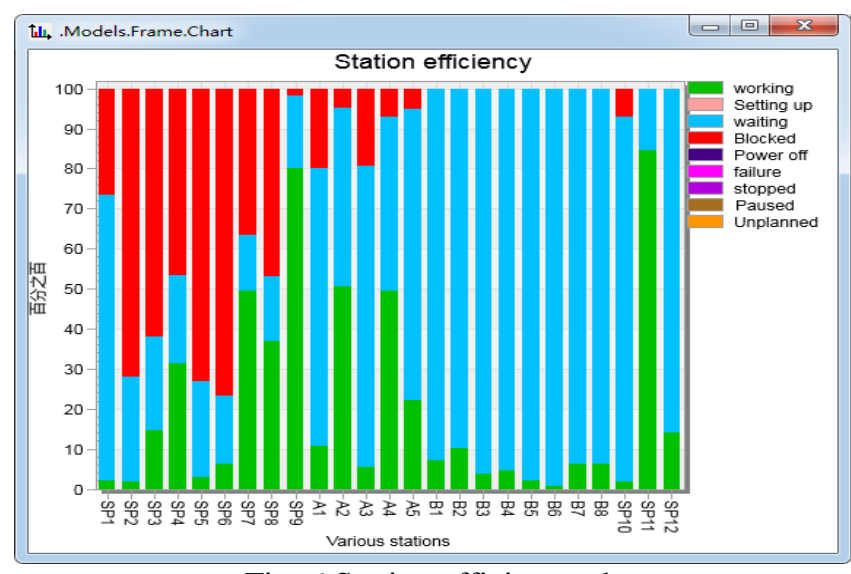

Fig. 6 Station efficiency chart

\section{EXPERIMENTAL DESIGN OPTIMIZATION MODEL}

Test Design DOE is a mathematical statistical method for arranging test and analysis test data. It is a technology based on statistics, product characteristics optimization, design and analysis, and is an important component of modern product design and quality management[6]. section. The experimental design has three basic principles: repetition, randomization and zoning. The orthogonal experimental design method and the factorial method are two commonly used experimental design methods. The test manager in the Plant Simulation software mainly uses the factorial method for testing.

In the production of marine products, unreasonable production is often caused by production bottlenecks caused by mismatches in order tasks and equipment configurations. The occurrence of these phenomena will lead to inefficient production, and the failure to complete the production plan according to the specified time will lead to delays in the production cycle, which will cause huge economic losses to 
the shipyard. Until now, most shipyards still rely on experienced managers to perform bottlenecks in production[7]. The accuracy of the judgement is very low, and bottlenecks are still happening frequently. Therefore, in order to prevent and eliminate the occurrence of bottlenecks in advance and improve the utilization rate of equipment, the experimental design method will be used to optimize the simulation model of the segmentation line.

By modeling the frontline and simulating the simulation, we can see from the data obtained in Figure 6 that there is a large production blockage in the station SP2-SP8, which seriously affects the production time of the subsequent stations, so that other stations exist. Long production waits for ineffective time, wasted time and equipment resources, and lengthens the ship's production cycle. There are two most common methods for eliminating production bottlenecks and reducing production blockage. The first one is to increase the parallel station to increase production, thereby reducing production blockage. The second is to increase the buffer station to make the blocking station. Production is buffered to ease the rate of blockage. In order to ensure lean shipbuilding, reduce inefficiency and shorten the construction period, we will optimize the SP2-SP8 station by adding parallel stations.

The SP2-SP8 station of the model in Fig. 3 is replaced by the ParallelProc station. The ParallelProc setting has $\mathrm{X}$ size and $\mathrm{Y}$ size, which means $\mathrm{M} \times \mathrm{N}$ stations. In the setting of the parallel station, One variable remains the same, and the number of parallel stations can be determined by changing another variable. The model is changed as shown in Figure 7.

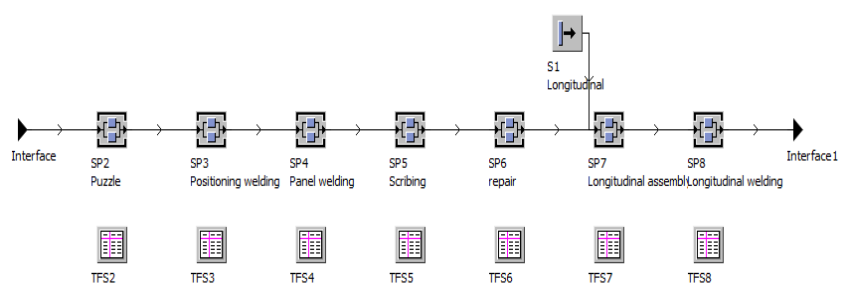

Fig. 7 Two-layer structure F1

Parallel stations are not set as much as possible. If there are too many workstations, they will waste equipment and human resources, and occupy too much space resources, resulting in unnecessary waste. If the number of parallel stations is insufficient, it cannot be Effectively reduce production time and reduce blocking rate. Therefore, the proper setting of the station is necessary, so it is necessary to test and verify the number of parallel stations by using the experimental design. Set the test manager to define the output value as the total time and the blocking rate of each station. The input value is the specific number of each parallel station, and the test upper and lower values are 5 and 1 , respectively. Is 1 .

It can be seen from the analysis of the results obtained by the test manager simulation that in the SP2-SP8 station, the increase of the parallel station of SP2-SP7 has little effect on reducing the blocking rate and shortening the production time, under the condition of limited funds and sites. It can be neglected, and the SP8 station increases the influence of the parallel station. The simulation result shows that when the parallel station is 2, it is the best working number. Therefore, the SP8 parallel station is set to 2, the others are still 1, and the total production time for modeling and simulation is 49:12:20:52, which is shortened by 3 days compared with the initial total production time.

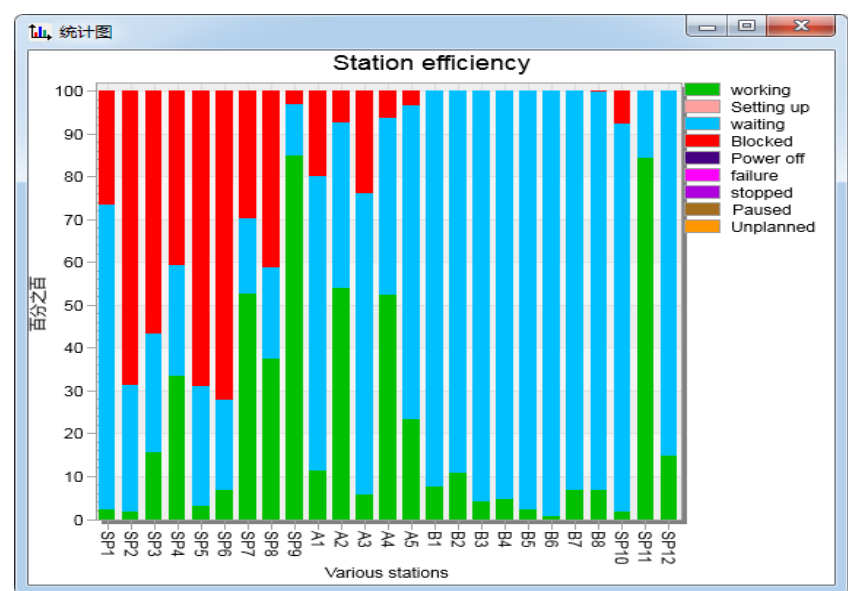

Fig. 8 Parallel station efficiency chart

According to Figure 8, after adding parallel stations, it can be found that although the total production time is improved and the blocking rate is relatively reduced, SP2-SP8 still has a large blocking rate and does not achieve the desired effect. Therefore, the method of increasing the cache station is used to further reduce the blocking rate. After the SP2-SP8 station, the buffer station Buffer is added separately, and the number of optimal cache stations is obtained by using the test manager simulation, As shown in Figure 9.

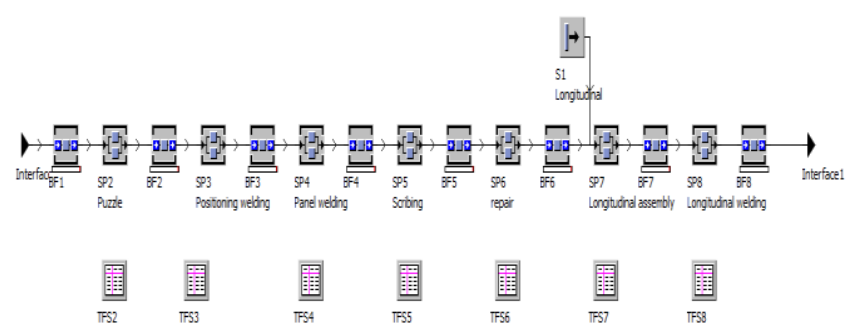

Fig. 9 F1 increases the simulation model after the cache station

The setting of the number of cache stations is the same as the setting of the parallel station. It cannot be set too much or too little, too much space is occupied, and the effect of reducing the cache is too small. Therefore, it is necessary to use the experimental design to optimize the test. The best number. Therefore, set the corresponding output value and input value, and define the test upper and lower values as 10 and 1, respectively, in increments of 1 . Analysis of all the test results obtained shows that setting the cache station after SP3, SP6 and SP8 has a greater impact on reducing the blocking rate and shortening the production time, so the other cache stations are removed, leaving only SP3, SP6 and SP8. After the setting of the cache station, the test manager simulation is performed again to solve the optimal number of stations, and the corresponding test upper and lower values are defined as 30 and 1 , respectively, in increments of 1 .

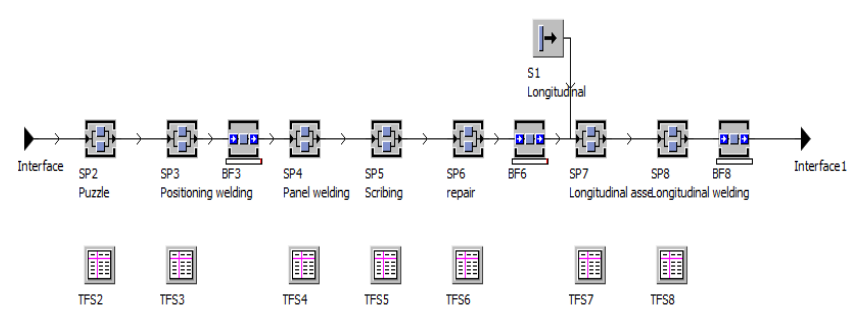

Fig. 10 F1 best cache station pipeline setting 
Through the simulation results of the test manager, we obtain the number of optimal cache stations when BF1, BF2, and $\mathrm{BF} 3$ are 25,20 , and 20 , respectively. In the same way, using the test manager to solve the optimal number of cache stations for SP1 and FA1 A1-A5 station simulation, the number of BF optimal stations after SP1 is 2, and the number of A1-A5 optimal stations is A1, A3, and A5 set the number of cache stations of the number 1,4 , and 2 , respectively. The specific situation is shown in Figure 11.
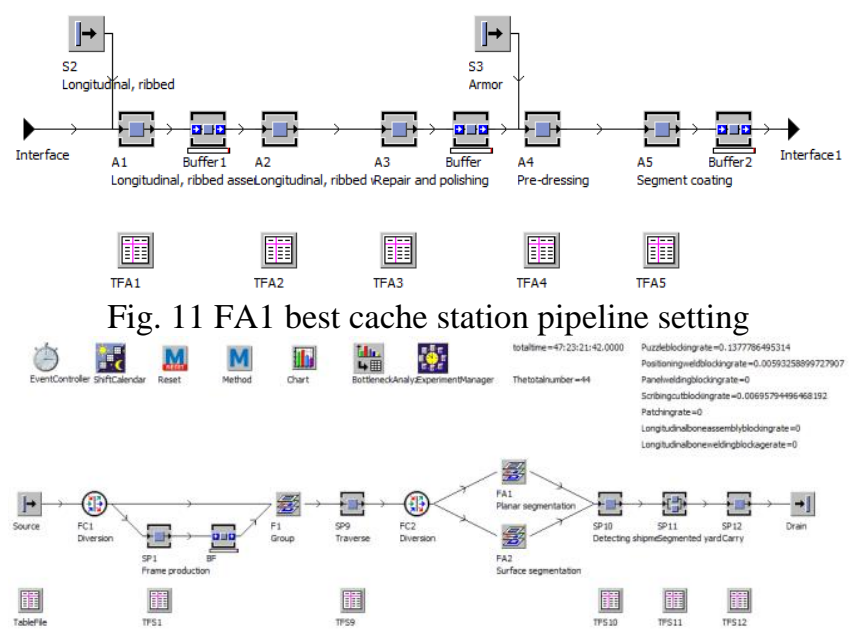

Fig. 12 Optimal cache station pipeline setting

Adding all the optimal number of stations to the simulation model and running the simulation to get the final total production time of $47: 23: 21: 42$, which is 4 days and a half less than the initial total production time of 52:12:59:21, and according to It can also be seen from Figure 13 that the production station blocking rate has also been greatly improved.

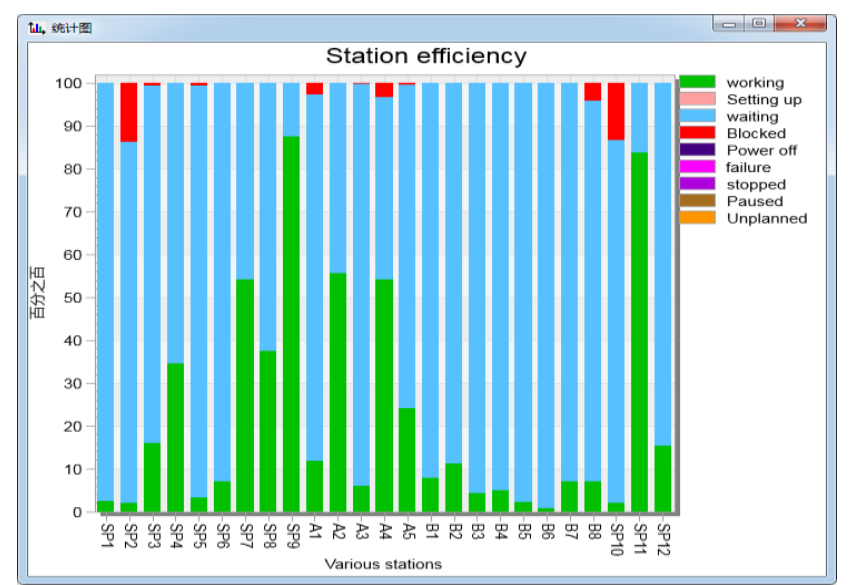

Fig. 13 Optimal cache station efficiency chart

We can see from Figure 13. The SP10 station has a certain blocking rate due to the unreasonable number of segmented yards. Originally, according to the shipyard production plan, eight sub-segments were prepared, so we need to increase the number of stacks appropriately. Also use the test manager to solve the optimal number, and find the optimal number of stacks is 12 , so that the final station efficiency is shown in Figure 14.

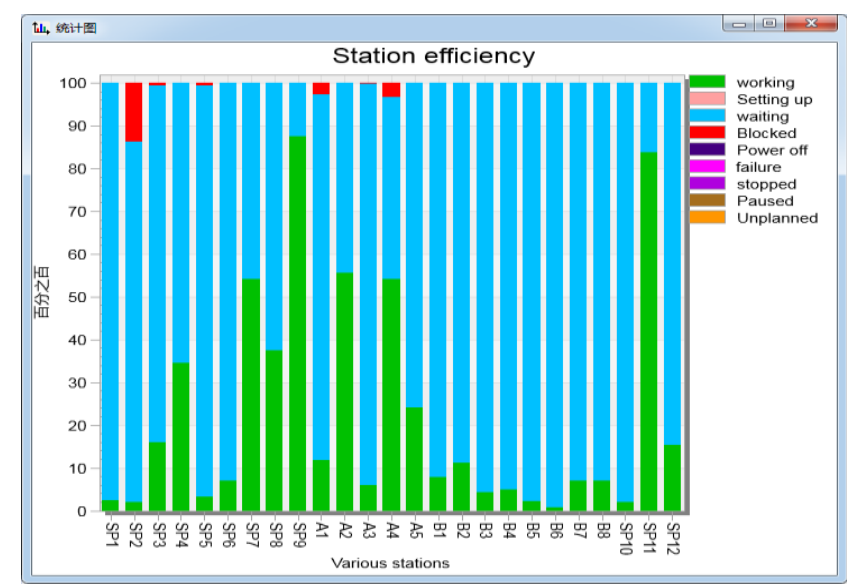

Fig. 14 Optimal station efficiency chart

\section{CONCLUSION}

By analyzing the importance of segmentation during shipbuilding and the current long construction period and high blockage rate of ship segmentation construction, Lean Shipbuilding knowledge is used to eliminate invalid time as much as possible. Using the simulation software Plant Simulation to model and simulate the segmentation construction process, in order to solve the problem of high blocking rate, replacing the parallel station and increasing the cache station, the problems in production are improved, and the DOE is designed through experiments. The calculation obtains the optimal solution, effectively reduces the waste of equipment resources, shortens the ship construction period, and alleviates production blockage.

\section{REFERENCES}

[1] Xu Shaoheng. The latest development of ship automation technology in twenty-first century[J]. Journal of Jiangsu University of Science and Technology(Natural Science Edition), 2014, 28(06): 511-517.

[2] Luo Wei, Liu Cungen, Zhang Wei, et al. A shipbuilding simulation system based on workshop units[J]. Shipbuilding of China, 2011, 52(03): 204-213.

[3] Wang Yue, Yao Wei, Jiang Zhiyong. Study on the simulation technology for the adjustment of shipbuilding lean scheme[J]. Journal of Jiangsu University of Science and Technology(Natural Science Edition), 2012, 26(06): 546-550.

[4] Wu Chao. Study on Virtual Shipyard Modeling Method and Application Based on Virtual Reality Technology[J]. Ship Engineering, 2015, 37(S1): 214-216.

[5] Xie Lei, Liu Cungen. simulation of ship construction process based on Em-plant[J]. Marine Technology, 2010, (04): 43-46.

[6] Jin Weijia,Liu Min,Lv Hongwei.Research on Process Simulation Based on Virtual Construction of Ships[J].Ship Standardization Engineer,2018,51(06):49-55.

[7] He Rui. Simulation and optimization of hull section construction production plan based on workshop group [D]. Zhejiang University, 2017.

Ma Xincheng, Student at Jiangsu University of Science and Technology. Graduate degree. Research direction for ship design and manufacturing

Zhang Guoqing, Student at Jiangsu University of Science and Technology. Graduate degree. Research direction for ship design and manufacturing

Zhang Chong, Student at Jiangsu University of Science and Technology. Graduate degree. Research direction for ship design and manufacturing 
Appendix

Tab. 1 Names and meanings of the controls

\begin{tabular}{|c|c|c|c|c|c|}
\hline category & $\begin{array}{c}\text { Name and } \\
\text { graphics }\end{array}$ & meaning & category & $\begin{array}{c}\text { Name and } \\
\text { graphics }\end{array}$ & meaning \\
\hline \multirow{7}{*}{$\begin{array}{l}\text { Physical } \\
\text { control }\end{array}$} & & $\begin{array}{l}\text { Source control for } \\
\text { simulating the feed port }\end{array}$ & \multirow{7}{*}{$\begin{array}{l}\text { Data } \\
\text { informat } \\
\text { ion } \\
\text { processi } \\
\text { ng } \\
\text { control }\end{array}$} & & $\begin{array}{l}\text { TableFile file, a table for } \\
\text { storing production data }\end{array}$ \\
\hline & & $\begin{array}{l}\text { Drain control for } \\
\text { simulation product } \\
\text { processing }\end{array}$ & & & $\begin{array}{l}\text { Program control, using } \\
\text { programming to control } \\
\text { simulation } \\
\end{array}$ \\
\hline & & $\begin{array}{c}\text { FlowControl control for } \\
\text { simulation of } \\
\text { analog/combination } \\
\end{array}$ & & & $\begin{array}{c}\text { Chart control for } \\
\text { analyzing the use of } \\
\text { workstation equipment }\end{array}$ \\
\hline & & $\begin{array}{l}\text { SingleProc control for } \\
\text { simulating analog } \\
\text { processing equipment }\end{array}$ & & & $\begin{array}{l}\text { Test manager, test design } \\
\text { tool }\end{array}$ \\
\hline & & $\begin{array}{l}\text { Assembly control for } \\
\text { simulating the assembly } \\
\text { process }\end{array}$ & & & $\begin{array}{l}\text { Bottleneck Analyzer, the } \\
\text { control to view the } \\
\text { bottleneck situation of } \\
\text { the station }\end{array}$ \\
\hline & & $\begin{array}{c}\text { ParallelProc control for } \\
\text { simulating multiple } \\
\text { parallel processing } \\
\text { devices }\end{array}$ & & & $\begin{array}{l}\text { Event controller, } \\
\text { controlling the running of } \\
\text { the simulation }\end{array}$ \\
\hline & & $\begin{array}{l}\text { Gantt Chart Wizard, a } \\
\text { tool for producing Gantt } \\
\text { charts }\end{array}$ & & & $\begin{array}{l}\text { Calendar, controls for } \\
\text { simulating production } \\
\text { and break times }\end{array}$ \\
\hline
\end{tabular}

Tab. 2 Segmentation production data for a type of ship

\begin{tabular}{|c|c|c|c|c|c|c|c|}
\hline Numbering & $\begin{array}{c}\text { Segme } \\
\text { ntation }\end{array}$ & Fragment & $\begin{array}{c}\text { Sheet } \\
\text { width / } \mathrm{m}\end{array}$ & $\begin{array}{c}\text { Puzzle } \\
\text { number }\end{array}$ & $\begin{array}{c}\text { Welding } \\
\text { meters }\end{array}$ & $\begin{array}{c}\text { Longitudinal } \\
\text { rice }\end{array}$ & Weight / \\
\hline 1 & D223 & Insole & 21.10 & 67.88 & 67.88 & 390.31 & 285.5639 \\
\hline 2 & D323 & Insole & 16.90 & 50.91 & 50.91 & 322.43 & 222.068 \\
\hline 3 & U017 & deck & 22.30 & 72 & 72 & 450 & 209.679 \\
\hline 4 & D331 & Insole & 16.90 & 50.91 & 50.91 & 322.43 & 210.4543 \\
\hline 5 & D231 & Insole & 21.10 & 67.88 & 67.88 & 390.31 & 267.311 \\
\hline 6 & D221 & Outer panel & 21.10 & 84.85 & 84.85 & 390.31 & 259.9822 \\
\hline 7 & Y222 & Vertical wall & 21.2 & 125.58 & 125.58 & 394.68 & 154.843 \\
\hline 8 & D321 & Outer panel & 16.90 & 67.88 & 67.88 & 322.43 & 204.2438 \\
\hline 9 & Y322 & Vertical wall & 21.2 & 125.58 & 125.58 & 394.68 & 178.8 \\
\hline 10 & S222 & deck & 4.68 & 17.97 & 17.97 & 89.85 & 268.8482 \\
\hline 11 & S222A & Inner shell & 20.64 & 107.82 & 107.82 & 377.37 & 268.8482 \\
\hline
\end{tabular}

